\title{
Isometric disks are holomorphic
}

\author{
Stergios M. Antonakoudis ${ }^{1}$
}

Received: 25 October 2015 / Accepted: 15 August 2016/ Published online: 5 October 2016 (C) The Author(s) 2016. This article is published with open access at Springerlink.com

\begin{abstract}
This paper shows that every totally-geodesic isometry from the unit disk to a finite-dimensional Teichmüller space for the intrinsic Kobayashi metric is either holomorphic or anti-holomorphic; in particular, it is a Teichmüller disk. Additionally, a similar result is proved for a large class of disk-rigid domains, which includes strictly convex domains, as well as finite-dimensional Teichmüller spaces.
\end{abstract}

\section{Contents}

1 Introduction . . . . . . . . . . . . . . . . . . . . . . . 1289

2 Preliminary results . . . . . . . . . . . . . . . . . . . . . 1291

3 Holomorphic rigidity for Teichmüller spaces . . . . . . . . . . . . . . . . . . . . 1294

4 The class of disk-rigid domains . . . . . . . . . . . . . . . . . . . . . . . 1297

References ........................... 1299

\section{Introduction}

Let $\mathbb{C} \mathbb{H}^{1}$ denote the unit disk $\Delta=\{z \in \mathbb{C}:|z|<1\}$ equipped with its Poincaré metric $|d z| /\left(1-|z|^{2}\right)$ and let $\mathcal{T}_{g, n}$ denote a finite-dimensional Teichmüller space equipped with its intrinsic Kobayashi metric, which is the

Stergios M. Antonakoudis

stergios@dpmms.cam.ac.uk

1 Department of Pure Mathematics and Mathematical Statistics, University of Cambridge, Cambridge, UK 
largest (Finsler) metric such that every holomorphic map $f: \mathbb{C H}^{1} \rightarrow \mathcal{T}_{g, n}$ is non-expanding: $\|d f\| \leq 1$.

An important feature of the Kobayashi metric of $\mathcal{T}_{g, n}$ is that every holomorphic map $f: \mathbb{C H}^{1} \rightarrow \mathcal{T}_{g, n}$, for which $d f$ is an isometry on tangent spaces, is totally geodesic: it sends real geodesics to real geodesics preserving their length. Moreover, there are such isometries through every point in every direction, known as Teichmüller disks.

Holomorphic rigidity for Teichmüller spaces Our main result in this paper is the following:

Theorem 1.1 Let $\mathcal{T}_{g, n}$ be a finite-dimensional Teichmüller space equipped with its intrinsic Kobayashi metric. Every totally geodesic isometry $f$ : $\mathbb{C H}^{1} \hookrightarrow \mathcal{T}_{g, n}$ is either holomorphic or anti-holomorphic; in particular, it is a Teichmüller disk.

Remark Theorem 1.1 settles a long standing problem in Teichmüller theory. ${ }^{1}$

The proof is geometric and rests on the idea of complexification; see Sect. 3. Informally, the theorem shows that the intrinsic Kobayashi metric of $\mathcal{T}_{g, n}$ determines its natural structure as a complex manifold.

As a corollary, we obtain the following general result about Teichmüller spaces.

Corollary 1.2 Let $\mathcal{T}_{g, n}, \mathcal{T}_{h, m}$ be two finite-dimensional Teichmüller spaces equipped with their intrinsic Kobayashi metric. Every totally geodesic isometry $f: \mathcal{T}_{g, n} \hookrightarrow \mathcal{T}_{h, m}$ is either holomorphic or anti-holomorphic.

We note that there are many holomorphic isometries $f: \mathcal{T}_{g, n} \hookrightarrow \mathcal{T}_{h, m}$ between Teichmüller spaces for their Kobayashi metric. [6, See discussion in Section 13.2.1].

Holomorphic rigidity for convex domains In addition to Theorem 1.1, we prove a similar result for a large class of disk-rigid domains, which include strictly convex domains, as well as finite-dimensional Teichmüller spaces. We discuss the general statement in Sect. 4; as a special case, we obtain:

Theorem 1.3 Let $\mathcal{B}_{1}, \mathcal{B}_{2}$ be two strictly convex bounded domains equipped with their intrinsic Kobayashi metric. Every totally geodesic isometry $f$ : $\mathcal{B}_{1} \hookrightarrow \mathcal{B}_{2}$ is either holomorphic or anti-holomorphic.

This result need not be true for convex domains in general. For example, the diagonal map $\delta(z)=(z, z)$ is a totally-real embedding $\delta: \mathbb{C H}^{1} \hookrightarrow$ $\mathbb{C H}^{1} \times \overline{\mathbb{C H}^{1}}$, which is a totally geodesic isometry for the Kobayashi metric. In particular, the result is not true for bounded symmetric domains with rank two or more.

\footnotetext{
${ }^{1}$ See problem 5.3 in [8].
} 


\section{Notes and references}

For an introduction to Teichmüller spaces and the Kobayashi metric on complex manifolds, we refer to $[9,12,14]$, respectively.

Royden proved that the Kobayashi metric of $\mathcal{T}_{g, n}$ coincides with its classical Teichmüller metric [19]. When $\operatorname{dim}_{\mathbb{C}} \mathcal{T}_{g, n}=1$, we can identify $\mathcal{T}_{g, n}$ equipped with its Kobayashi-Teichmüller metric with the unit disk $\mathbb{C} \mathbb{H}^{1}$ equipped with its Poincaré metric. In particular, the first instance of Theorem 1.1 is implicit in the natural isomorphism $\left.\operatorname{Aut}\left(\mathbb{C H}^{1}\right) \cong \operatorname{Isom}^{+}(\mathbb{C H})^{1}\right)$ between the group of holomorphic automorphisms of the unit disk and the group of orientationpreserving isometries of its Poincaré metric, which is classically known as the Schwarz-Pick lemma [14, Section 2].

There is a natural action of $\mathrm{SL}_{2}(\mathbb{R})$ on the sphere bundle of unit-area quadratic differentials $Q_{1} \mathcal{T}_{g, n}$ over $\mathcal{T}_{g, n}$, so that every orbit projects to a holomorphic totally geodesic isometry $\mathbb{C H}^{1} \cong \mathrm{SO}_{2}(\mathbb{R}) \backslash \mathrm{SL}_{2}(\mathbb{R}) \hookrightarrow \mathcal{T}_{g, n}$, which is known as a Teichmüller disk. It is a classical result (see Theorem 2.1 in Section 2) that every holomorphic isometry $\mathbb{C H}{ }^{1} \hookrightarrow \mathcal{T}_{g, n}$ into a finite-dimensional Teichmüller space is a Teichmüller disk. However, neither this result, nor Theorem 1.1 remain true for infinite dimensional Teichmüller spaces, since they contain [5] holomorphic isometric copies of the bi-disk, for which these two results are not true.

A complex analytic proof that totally geodesic disks are holomorphic for strictly convex domains with $\mathrm{C}^{3}$-smooth boundary appears in [10]. Theorem 1.3 gives an optimal result for maps between convex domains. In a follow-up paper (to appear), we show that Teichmüller spaces with $\operatorname{dim}_{\mathbb{C}} \mathcal{I}_{g, n} \geq$ 2 cannot be realised as convex domains.

\section{Preliminary results}

The Kobayashi metric [14] Let $\mathcal{B} \subset \mathbb{C}^{N}$ be a bounded domain. The intrinsic Kobayashi metric of $\mathcal{B}$ is the largest complex Finsler metric such that every holomorphic map $f: \mathbb{C H}^{1} \rightarrow \mathcal{B}$ is non-expanding: $\|d f\|_{\mathcal{B}} \leq 1$. It determines both a family of norms $\|\cdot\|_{\mathcal{B}}$ on tangent spaces and a distance function $d_{\mathcal{B}}(\cdot, \cdot)$ on pairs of points.

We recall that the Schwarz-Pick lemma [14, Section 2] implies that every holomorphic map $f: \mathbb{C H}^{1} \rightarrow \mathbb{C H} \mathbb{H}^{1}$ is non-expanding. The Kobayashi metric provides a natural generalisation - it has the fundamental property that every holomorphic map between complex domains is non-expanding. In particular, a holomorphic automorphism is always an isometry and the Kobayashi metric of a complex domain depends only on its structure as a complex manifold. 


\section{Examples}

(1) $\mathbb{C H}^{1} \cong\{z \in \mathbb{C}:|z|<1\}$ with its Poincaré metric $|d z| /\left(1-|z|^{2}\right)$ coincides with the Kobayashi metric, by Schwarz's lemma. More generally, the Kobayashi metric of the ball $\mathbb{C} \mathbb{H}^{N} \cong\left\{\left(z_{i}\right)_{i=1}^{N} \in \mathbb{C}^{N}: \sum_{i=1}^{N}\left|z_{i}\right|^{2}<1\right\}$ coincides with its complete invariant (Kähler) metric of constant holomorphic curvature -4. See [14, Example 3.1.24].

(2) The Kobayashi metric of the bi-disk $\mathbb{C H}^{1} \times \overline{\mathbb{C H}^{1}}$ is the maximum metric of the two factors. It is a complex Finsler metric, which is not Hermitian. The distance function is given by $d_{\mathbb{C H}^{1} \times \overline{\mathbb{C H}}}\left(\left(z_{1}, z_{2}\right),\left(w_{1}, w_{2}\right)\right)=$ $\max \left\{d_{\mathbb{C H}^{1}}\left(z_{1}, w_{1}\right), d_{\mathbb{C H}^{1}}\left(z_{2}, w_{2}\right)\right\}$ for all points $\left(z_{1}, z_{2}\right),\left(w_{1}, w_{2}\right) \in$ $\mathbb{C H}^{1} \times \overline{\mathbb{C H}^{1}}$. See [14, Corollary 3.1.10].

(3) The Kobayashi metric of $\mathcal{T}_{g, n}$ coincides with its classical Teichmüller metric, which endows $\mathcal{T}_{g, n}$ with the structure of a complete geodesic metric space [19]. We discuss this example in more detail below.

Complex geodesics Let $\gamma_{\mathbb{C}}: \mathbb{C H}^{1} \rightarrow \mathcal{B}$ be a holomorphic (or antiholomorphic) map; we call $\gamma_{\mathbb{C}}$ a complex geodesic if it is locally distance preserving for the Kobayashi metric; or equivalently, if it is a totally geodesic isometry: $\gamma_{\mathbb{C}}$ sends real geodesics to real geodesics preserving their length. We note that, in this case, the map given by $\gamma(t)=\gamma_{\mathbb{C}}\left(e^{i \theta} \tanh (t)\right)$ for $t \in \mathbb{R}$, defines a complete, unit-speed, real geodesic line, for every $\theta \in \mathbb{R} / 2 \pi \mathbb{Z}$. When it is clear from the context, we will often identify the real and complex geodesics with their image in $\mathcal{B}$.

Teichmüller space $[9,12]$ Let $\Sigma_{g, n}$ be a connected, oriented surface of genus $g$ and $n$ punctures and $\mathcal{T}_{g, n}$ denote the Teichmüller space of Riemann surfaces marked by $\Sigma_{g, n}$. A point in $\mathcal{T}_{g, n}$ is specified by an equivalence class ${ }^{2}$ of orientation preserving homeomorphisms $\phi: \Sigma_{g, n} \rightarrow X$, where $X$ is a Riemann surface of finite type.

Teichmüller space $\mathcal{T}_{g, n}$ is the orbifold universal cover of the moduli space of Riemann surfaces $\mathcal{M}_{g, n}$ and is naturally a complex manifold with dimension $3 g-3+n$. It is known [3] that Teichmüller space can be realized as a contractible bounded domain of holomorphy $\mathcal{T}_{g, n} \subset \mathbb{C}^{3 g-3+n}$ by the Bers embeddings.

Teichmüller metric For each $X \in \mathcal{T}_{g, n}$, we let $Q(X)$ denote the space of holomorphic quadratic differentials $q=q(z)(d z)^{2}$ on $X$ with finite total mass: $\|q\|_{1}=\int_{X}|q(z) \| d z|^{2}<+\infty$, which means that $q$ has at worse simple poles at the punctures of $X$. The tangent and cotangent spaces to Teichmüller space at $X \in \mathcal{T}_{g, n}$ are described in terms of the natural pairing $(q, \mu) \mapsto \int_{X} q \mu$

\footnotetext{
2 Two marked Riemann surfaces $\phi: \Sigma_{g, n} \rightarrow X, \psi: \Sigma_{g, n} \rightarrow Y$ are equivalent if $\psi \circ \phi^{-1}$ : $X \rightarrow Y$ is isotopic to a holomorphic bijection.
} 
between the space $Q(X)$ and the space $M(X)$ of $L^{\infty}$-measurable Beltrami differentials on $X$; in particular, the tangent $T_{X} \mathcal{T}_{g, n}$ and cotangent $T_{X}^{*} \mathcal{T}_{g, n}$ spaces are naturally isomorphic to $M(X) / Q(X)^{\perp}$ and $Q(X)$, respectively.

The Teichmüller-Kobayashi metric on $\mathcal{T}_{g, n}$ is given by norm duality on the tangent space $T_{X} \mathcal{T}_{g, n}$ from the norm $\|q\|_{1}=\int_{X}|q|$ on the cotangent space $Q(X)$ at $X$. The corresponding distance function is given by the formula $d_{\mathcal{T}_{g, n}}(X, Y)=\inf \frac{1}{2} \log K(\phi)$ and measures the minimal dilatation $K(\phi)$ of a quasiconformal map $\phi: X \rightarrow Y$ respecting their markings.

The Teichmüller metric is complete and coincides with the Kobayashi metric of $\mathcal{T}_{g, n}$ as a complex manifold [19]. In particular, it has the remarkable property that every holomorphic map $f: \mathbb{C H}^{1} \rightarrow \mathcal{T}_{g, n}$ is non-expanding: $\|d f\|_{\mathcal{T}_{g, n}} \leq 1$. Holomorphic disks We summarise below the main results about holomorphic disks in Teichmüller space which we shall employ in the proof of Theorem 1.1.

Complex geodesics in Teichmüller space are abundant: there is one through every point in $\mathcal{T}_{g, n}$ in every complex direction, classically known as Teichmüller disks.

In particular, every complex geodesic $\gamma_{\mathbb{C}}: \mathbb{C H}^{1} \hookrightarrow \mathcal{T}_{g, n}$ gives rise to a unit-speed real geodesic $\gamma: \mathbb{R} \hookrightarrow \mathcal{T}_{g, n}$ by $\gamma(t)=\gamma_{\mathbb{C}}(\tanh (t))$, for $t \in \mathbb{R}$. Conversely, every unit-speed real geodesic $\gamma: \mathbb{R} \hookrightarrow \mathcal{T}_{g, n}$ extends uniquely to a complex geodesic $\gamma_{\mathbb{C}}: \Delta \cong \mathbb{C H}^{1} \hookrightarrow \mathcal{T}_{g, n}$ such that $\gamma(t)=\gamma_{\mathbb{C}}(\tanh (t))$, for $t \in \mathbb{R}$.

The following classical result characterises the holomorphic disks in Teichmüller space which are complex geodesics for the Kobayashi metric. See [4], for a simple proof based on Slodkowski's theorem [20].

Theorem 2.1 Let $f: \Delta \cong \mathbb{C H}^{1} \rightarrow \mathcal{T}_{g, n}$ be a holomorphic map with $\left\|f^{\prime}(0)\right\|_{\mathcal{T}_{g, n}}=1$, then $f$ is a totally geodesic isometry for the Kobayashi metric; in particular, it is a Teichmüller disk.

The following important result, which is a consequence of Sullivan's rigidity theorem [21], shows that there are no non-trivial holomorphic families of essentially proper (see Sect. 4) holomorphic disks in Teichmüller space. See [22, Theorem 1] for a simple proof and [16] for further applications and related ideas.

Theorem 2.2 Let $\left\{f_{t}\right\}_{t \in \Delta}$ be a holomorphic family of holomorphic maps $f_{t}$ : $\Delta \cong \mathbb{C H} \mathbb{H}^{1} \rightarrow \mathcal{T}_{g, n}$ and $B_{f_{0}} \subset \partial \Delta$ denote the set of bounded rays of $f_{0}$, i.e. $B_{f_{0}}=\left\{e^{i \theta} \in \partial \Delta: \sup _{t \in[0,1)} d_{\mathcal{T}_{g, n}}\left(f_{0}(0), f_{0}\left(t e^{i \theta}\right)\right)<+\infty\right\}$. If $\partial \Delta \backslash B_{f_{0}}$ has positive (Lebesgue) measure, then the family is trivial: $f_{t}=f_{0}$ for all $t \in \Delta$.

We discuss a more general class of disk-rigid domains satisfying Theorem 2.2 and formulate a generalisation of Theorem 1.1 in Sect. 4. 


\section{Holomorphic rigidity for Teichmüller spaces}

In this section we prove:

Theorem 3.1 Every totally geodesic isometry $f: \mathbb{C H}^{1} \hookrightarrow \mathcal{T}_{g, n}$ for the Kobayashi metric is either holomorphic or anti-holomorphic. In particular, it is a Teichmüller disk.

The proof of the theorem rests on the idea of complexification and leverages the following two facts. Firstly, a complete real geodesic in $\mathcal{T}_{g, n}$ is contained in a unique holomorphic Teichmüller disk; and secondly, Theorem 2.2: a holomorphic family $\left\{f_{t}\right\}_{t \in \Delta}$ of essentially proper holomorphic maps $f_{t}: \mathbb{C H}^{1} \rightarrow \mathcal{T}_{g, n}$ is trivial: $f_{t}=f_{0}$.

Outline of the proof Let $\gamma \subset \mathbb{C H}{ }^{1}$ be a complete (real) geodesic and denote by $\gamma_{\mathbb{C}} \subset \mathbb{C H}^{1} \times \overline{\mathbb{C H}^{1}}$ the maximal holomorphic extension of the diagonal embedding of $\gamma$ to the bi-disk. We note that $\gamma_{\mathbb{C}} \cong \mathbb{C H}^{1}$ and we define $\left.F\right|_{\gamma_{\mathbb{C}}}$ to be the unique holomorphic extension of $\left.f\right|_{\gamma}$ which is a Teichmüller disk.

Applying this construction to all real geodesics in $\mathbb{C H}^{1}$, we deduce that the isometry $f: \mathbb{C H}^{1} \rightarrow \mathcal{T}_{g, n}$ extends to a map $F: \mathbb{C H}^{1} \times \overline{\mathbb{C H}^{1}} \rightarrow \mathcal{T}_{g, n}$ such that $f(z)=F(z, z)$, for $z \in \Delta \cong \mathbb{C} \mathbb{H}^{1}$, and prove that $F$ is holomorphic. Using that $f$ is a totally geodesic isometry, we show that the map $F$ is essentially proper and conclude, by Theorem 2.2, that either $F(z, w)=F(z, z)$ or $F(z, w)=F(w, w)$ for $(z, w) \in \mathbb{C H}^{1} \times \overline{\mathbb{C} \mathbb{H}^{1}}$.

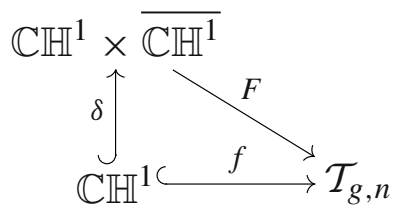

We start with some preliminary constructions.

The totally real diagonal Let $\overline{\mathbb{C H}^{1}}$ be the complex hyperbolic line with its conjugate complex structure. The identity map is a canonical anti-holomorphic isomorphism $\mathbb{C H}^{1} \cong \overline{\mathbb{C H}^{1}}$ and its graph is a totally real embedding $\delta$ : $\mathbb{C H} \mathbb{H}^{1} \hookrightarrow \mathbb{C H}^{1} \times \overline{\mathbb{C H}^{1}}$, given by $\delta(z)=(z, z)$ for $z \in \Delta \cong \mathbb{C H}^{1}$. We call $\delta\left(\mathbb{C} \mathbb{H}^{1}\right)$ the totally real diagonal.

Geodesics and graphs of reflections Let $\mathcal{G}$ denote the set of all real, unoriented, complete geodesics $\gamma \subset \mathbb{C H}^{1}$. In order to describe their maximal holomorphic extensions $\gamma_{\mathbb{C}} \subset \mathbb{C} \mathbb{H}^{1} \times \overline{\mathbb{C H}}^{1}$, such that $\left.\gamma_{\mathbb{C}} \cap \delta(\mathbb{C H})^{1}\right)=\delta(\gamma)$, it is convenient to parametrize $\mathcal{G}$ in terms of the set $\mathcal{R}$ of hyperbolic reflections of $\mathbb{C H} \mathbb{H}^{1}$ —or equivalently, the set of anti-holomorphic involutions of $\mathbb{C H}{ }^{1}$. The map that associates a reflection $r \in \mathcal{R}$ with the set $\gamma=\operatorname{Fix}(r) \subset \mathbb{C H}^{1}$ of its fixed points gives a bijection between $\mathcal{R}$ and $\mathcal{G}$. 
Let $r \in \mathcal{R}$ and denote its graph by $\Gamma_{r} \subset \mathbb{C H}^{1} \times \overline{\mathbb{C H}^{1}}$; there is a natural holomorphic isomorphism $\mathbb{C H}^{1} \cong \Gamma_{r}$, given by $z \mapsto(z, r(z))$ for $z \in \Delta \cong$ $\mathbb{C H}^{1}$. We note that $\Gamma_{r}$ is the maximal holomorphic extension $\gamma_{\mathbb{C}}$ of the geodesic $\gamma=\operatorname{Fix}(r)$ to the bi-disk and it is uniquely determined by the property $\gamma_{\mathbb{C}} \cap$ $\delta\left(\mathbb{C} \mathbb{H}^{1}\right)=\delta(\gamma)$.

The foliation by graphs of reflections The union of the graphs of reflections $\bigcup_{r \in \mathcal{R}} \Gamma_{r}$ gives rise to a (singular) foliation of $\mathbb{C} \mathbb{H}^{1} \times \overline{\mathbb{C H}^{1}}$ with holomorphic leaves $\Gamma_{r}$ parametrized by the set $\mathcal{R}$. We have $\Gamma_{r} \cap \delta\left(\mathbb{C} \mathbb{H}^{1}\right)=\delta(\operatorname{Fix}(r))$ for all $r \in \mathcal{R}$, and

$$
\Gamma_{r} \cap \Gamma_{s}=\delta(\operatorname{Fix}(r) \cap \operatorname{Fix}(s))
$$

which is either empty or a single point for all $r, s \in \mathcal{R}$ with $r \neq s$. In particular, the foliation is smooth in the complement of the totally real diagonal $\delta\left(\mathbb{C} \mathbb{H}^{1}\right)$.

We emphasize that the following simple observation plays a key role in the proof of the theorem. For all $r \in \mathcal{R}$ :

$$
(z, w) \in \Gamma_{r} \Longleftrightarrow(w, z) \in \Gamma_{r}
$$

Geodesics and the Klein model The Klein model gives a real-analytic identification $\mathbb{C H}^{1} \cong \mathbb{R H}^{2} \subset \mathbb{R}^{2}$ with an open disk in $\mathbb{R}^{2}$. It has the nice property that the hyperbolic geodesics are affine straight lines intersecting the disk [18].

Remark The holomorphic foliation by graphs of reflections defines a canonical complex structure in a neighborhood of the zero section of the tangent bundle of $\mathbb{R} H^{2}$.

The description of geodesics in the Klein model is convenient in the light of the following theorem.

Theorem 3.2 (S. Bernstein) Let $f:[0,1]^{2} \rightarrow \mathbb{C}$ be a function on the square $[0,1]^{2} \subset \mathbb{R}^{2} \subset \mathbb{C}^{2}$ and let $E \subset \mathbb{C}$ denote a fixed ellipse with foci at 0 and 1. If for every vertical and every horizontal unit line segment $\ell \cong[0,1]$, there is a holomorphic function $F_{\ell}: E_{\ell} \rightarrow \mathbb{C}$, defined on the ellipse $E_{\ell}$ with foci at the end-points of $\ell$ and congruent to $E$, that agrees with $f$ along the line segment $\ell:\left.\left.f\right|_{\ell} \cong F_{\ell}\right|_{\ell}$, then the function $f$ has a (unique germ of) holomorphic extension in a neighborhood of $[0,1]^{2}$ in $\mathbb{C}^{2}$.

See [1] for a simple proof of the theorem; ${ }^{3}$ and [13, Theorem 5.6.5], [2, Theorem 3.4 and Corollary 3.6] for a more general result and discussion of related ideas.

We use Theorem 3.2 to prove:

\footnotetext{
3 Bernstein's clever proof was to consider this as problem on the torus obtained from a branched covering $S^{1} \times S^{1} \rightarrow[0,1] \times[0,1]$ with Galois group $\mathbb{Z} / 2 \mathbb{Z} \times \mathbb{Z} / 2 \mathbb{Z}$, and use the symmetries of the pull back function to show that its Fourier coefficients vanish outside the first quadrant.
} 
Lemma 3.3 Every totally geodesic isometry $f: \mathbb{C H}^{1} \hookrightarrow \mathcal{T}_{g, n}$ admits a unique holomorphic extension in a neighborhood of the totally real diagonal $\delta\left(\mathbb{C H} \mathbb{H}^{1}\right) \subset \mathbb{C H}^{1} \times \overline{\mathbb{C H}^{1}}$.

Proof of 3.3 Using the fact that analyticity is a local property and the description of geodesics in the Klein model of $\mathbb{R} \mathbb{H}^{2}$, we can assume-without loss of generality - that the map $f$ is defined in a neighborhood of the unit square $[0,1]^{2}$ in $\mathbb{R}^{2}$ and has the property that its restriction on every horizontal and vertical line segment $\ell \cong[0,1]$ is a real-analytic parametrization of a Teichmüller geodesic segment. Moreover, we can also assume that the lengths of all these segments, measured in the Teichmüller metric, are uniformly bounded from above and from below away from zero.

Since every segment of a Teichmüller geodesic extends to a holomorphic Teichmüller disk in $\mathcal{T}_{g, n}$, there exists an ellipse $E \subset \mathbb{C}$ with foci at 0,1 such that the restrictions $\left.f\right|_{\ell}$ extend to holomorphic maps $F_{\ell}: E \rightarrow \mathcal{T}_{g, n}$ for all horizontal and vertical line segments $\ell \cong[0,1]$ of $[0,1]^{2}$. Hence, the proof of the lemma follows from Theorem 3.2.

\subsection{Proof of Theorem 3.1}

Let $f: \mathbb{C H}^{1} \hookrightarrow \mathcal{T}_{g, n}$ be a totally geodesic isometry. Applying Lemma 3.3, we deduce that $f$ has a unique holomorphic extension in a neighborhood of the totally real diagonal $\delta\left(\mathbb{C H} \mathbb{H}^{1}\right) \subset \mathbb{C H} \mathbb{H}^{1} \times \overline{\mathbb{C H}} \mathbb{H}^{1}$. We will show that $f$ extends to a holomorphic map from $\mathbb{C H}^{1} \times \overline{\mathbb{C H} H^{1}}$ to $\mathcal{T}_{g, n}$.

We start by defining a new map $F: \mathbb{C H} \mathbb{H}^{1} \times \overline{\mathbb{C} \mathbb{H}^{1}} \rightarrow \mathcal{T}_{g, n}$, satisfying:

1. $F(z, z)=f(z)$ for all $z \in \Delta \cong \mathbb{C} \mathbb{H}^{1}$.

2. $\left.F\right|_{\Gamma_{r}}$ is the unique holomorphic extension of $\left.f\right|_{\mathrm{Fix}(r)}$ for all $r \in \mathcal{R}$.

Let $r \in \mathcal{R}$ be a reflection. There is a unique (holomorphic) Teichmüller disk $\phi_{r}: \mathbb{C H}^{1} \hookrightarrow \mathcal{T}_{g, n}$ such that the intersection $\phi_{r}\left(\mathbb{C H} H^{1}\right) \cap f\left(\mathbb{C H}{ }^{1}\right) \subset \mathcal{T}_{g, n}$ contains the Teichmüller geodesic $f(\operatorname{Fix}(r))$ and $\phi_{r}(z)=f(z)$ for all $z \in$ $\operatorname{Fix}(r)$.

We define $F$ by $F(z, r(z))=\phi_{r}(z)$ for $z \in \mathbb{C H}^{1}$ and $r \in \mathcal{R}$; Eq. (3.1) shows that $F$ is well-defined and satisfies conditions (1) and (2) above.

We claim that $F: \mathbb{C H}^{1} \times \overline{\mathbb{C H}^{1}} \rightarrow \mathcal{T}_{g, n}$ is the unique holomorphic extension of $f: \mathbb{C H}^{1} \hookrightarrow \mathcal{T}_{g, n}$ such that $F(z, z)=f(z)$ for $z \in \mathbb{C H}^{1}$.

Proof of claim We note that the restriction of $F$ on the totally real diagonal $\delta\left(\mathbb{C H}^{1}\right)$ agrees with $f$ and that there is a unique germ of holomorphic maps near $\delta\left(\mathbb{C H} \mathbb{H}^{1}\right)$ whose restriction on $\delta\left(\mathbb{C H} \mathbb{H}^{1}\right)$ coincides with $f$. Let us fix an element of this germ $\tilde{F}$ defined on a neighborhood $U \subset \mathbb{C H}^{1} \times \overline{\mathbb{C H} \mathbb{H}^{1}}$ of $\delta\left(\mathbb{C H} \mathbb{H}^{1}\right)$. For every $r \in \mathcal{R}$, the restrictions of $F$ and $\tilde{F}$ on the intersection $U_{r}=U \cap \Gamma_{r}$ 
are holomorphic and equal along the real-analytic arc $U_{r} \cap \delta\left(\mathbb{C H} \mathbb{H}^{1}\right) \subset U_{r}$; hence they are equal on $U_{r}$. Since $\mathbb{C H}^{1} \times \overline{\mathbb{C H}^{1}}=\bigcup_{r \in \mathcal{R}} \Gamma_{r}$, we conclude that $\left.F\right|_{U}=\tilde{F}$ and, in particular, $F$ is holomorphic near the totally real diagonal $\delta\left(\mathbb{C H} \mathbb{H}^{1}\right)$. Since, in addition to this, $F$ is holomorphic along all the leaves $\Gamma_{r}$ of the foliation, we deduce ${ }^{4}$ that it is holomorphic at all points of $\mathbb{C H}^{1} \times \overline{\mathbb{C H}}^{1}$.

In order to complete the proof, we recall the key observation (3.2) as follows: the points $(z, w)$ and $(w, z)$ are always contained in the same leaf $\Gamma_{r}$ of the foliation; and since the restriction of $F$ on every leaf $\Gamma_{r}$ is a Teichmüller disk, we conclude that: $d_{\mathcal{T}_{g, n}}(F(z, w), F(w, z))=d_{\mathbb{C H}^{1}}(z, w)$ for all $z, w \in \Delta \cong$ $\mathbb{C H}^{1}$. In particular, at least one of $F\left(\rho e^{i \theta}, 0\right)$ and $F\left(0, \rho e^{i \theta}\right)$ diverges in $\mathcal{T}_{g, n}$, as $\rho \rightarrow 1$, for every $\theta \in \mathbb{R} / 2 \pi \mathbb{Z}$.

It follows that there is a subset $I \subset \mathbb{R} / 2 \pi \mathbb{Z}$ with (positive) Lebesgue measure at least $1 / 2$, so that either $F\left(\rho e^{i \theta}, 0\right)$ diverges as $\rho \rightarrow 1$, for all $\theta \in I$, or $F\left(0, \rho e^{i \theta}\right)$ diverges as $\rho \rightarrow 1$, for all $\theta \in I$. If we assume that the former of the two is true, we deduce from Theorem 2.2 that the holomorphic family $\{F(z, \bar{w})\}_{w \in \Delta}$ of holomorphic maps $F(\cdot, \bar{w}): \Delta \cong \mathbb{C H}^{1} \rightarrow \mathcal{T}_{g, n}$ is trivial; therefore, $F(z, 0)=F(z, z)=f(z)$ for all $z \in \Delta$ and, in particular, $f$ is holomorphic. If we assume that the latter of the two is true, we similarly deduce that $F(0, z)=F(z, z)=f(z)$ for all $z \in \Delta$ and, in particular, $f$ is anti-holomorphic.

\section{The class of disk-rigid domains}

In this section we formulate a general theorem that applies to a large class of bounded domains, which we apply to deduce Corollary 1.2 and Theorem 1.3.

Let $\mathcal{B} \subset \mathbb{C}^{N}$ be a bounded domain and $f: \Delta \rightarrow \mathcal{B}$ a holomorphic map. We call the map $f$ essentially proper if $\partial \Delta \backslash B_{f}$ has positive (Lebesgue) measure, where $B_{f}$ denotes the set of bounded rays, i.e. $B_{f}=\left\{e^{i \theta} \in\right.$ $\left.\partial \Delta: \sup _{t \in[0,1)} d_{\mathcal{B}}\left(f(0), f\left(t e^{i \theta}\right)\right)<+\infty\right\}$.

Definition 4.1 A bounded domain $\mathcal{B} \subset \mathbb{C}^{N}$ is disk-rigid, if it satisfies:

1. every unit-speed geodesic $\gamma: \mathbb{R} \hookrightarrow \mathcal{B}$, for the Kobayashi metric, extends to a complex geodesic $\gamma_{\mathbb{C}}: \Delta \cong \mathbb{C H}^{1} \hookrightarrow \mathcal{B}$ such that $\gamma(t)=\gamma_{\mathbb{C}}(\tanh (t))$, for $t \in \mathbb{R}$,

2. every holomorphic family $\left\{f_{t}\right\}_{t \in \Delta}$ of holomorphic maps $f_{t}: \Delta \cong \mathbb{C H}^{1} \rightarrow$ $\mathcal{B}$, with $f_{0}$ an essentially proper map, is trivial ie. $f_{t}=f_{0}$ for all $t \in \Delta$.

\footnotetext{
${ }^{4}$ For a simple proof of this, using the power series expansion of $F$ at $(0,0) \in \mathbb{C H}^{1} \times \overline{\mathbb{C H}^{1}}$, see [11, Lemma 2.2.11].
} 


\section{Examples}

1. Teichmüller spaces $\mathcal{T}_{g, n}$ of finite dimension are disk-rigid. See Sect. 2, Theorems 2.1, 2.2.

2. The bi-disk $\mathbb{C H}^{1} \times \overline{\mathbb{C H}^{1}}$ is a convex domain that is not disk-rigid. A bounded symmetric domain $\mathcal{B} \subset \mathbb{C}^{N}$ is disk-rigid if and only if it has rank one: $\mathcal{B} \cong \mathbb{C H}^{N}$.

3. All strictly convex bounded domains $\mathcal{B} \subset \mathbb{C}^{N}$ are disk-rigid. We recall that a domain $\mathcal{B} \subset \mathbb{C}^{N}$ is strictly convex if $\{t \cdot P+(1-t) \cdot Q: t \in(0,1)\} \subset \mathcal{B}$ for every pair of distinct points $P \neq Q$ in the closure $\overline{\mathcal{B}} \subset \mathbb{C}^{N}$. See [17].

The proof of Theorem 3.1 in Sect. 3 used only those features of $\mathcal{T}_{g, n}$ captured in the definition of a disk-rigid domain. In particular, the following result follows as well.

Theorem 4.2 Let $\mathcal{B} \subset \mathbb{C}^{N}$ be a disk-rigid domain. Every totally geodesic isometry $f: \mathbb{C H}^{1} \hookrightarrow \mathcal{B}$ for the Kobayashi metric is either holomorphic or anti-holomorphic.

We also have the following generalisation, which implies Corollary 1.2 and Theorem 1.3. The proof follows from Theorem 4.2 and Weyl's regularity lemma.

Theorem 4.3 Let $\mathcal{B}_{1}, \mathcal{B}_{2}$ be two complete disk-rigid domains for the Kobayashi metric. Every totally geodesic isometry $f: \mathcal{B}_{1} \hookrightarrow \mathcal{B}_{2}$ is either holomorphic or anti-holomorphic.

Proof In a sufficiently small neighborhood of a point, the Kobayashi metric is bi-Lipschitz to a Hermitian metric [14]. It follows that a totally geodesic isometry $f: \mathcal{B}_{1} \hookrightarrow \mathcal{B}_{2}$ is locally Lipschitz and hence it is differentiable at almost all points of $\mathcal{B}_{1}$, by Rademacher's theorem (see Theorem 3.1.6 in [7]).

Let $p \in \mathcal{B}_{1}$ such that the (real) linear map $d f_{p}: T_{p} \mathcal{B}_{1} \rightarrow T_{p} \mathcal{B}_{2}$ exists. Using Theorem 4.2, we conclude that $f$ sends complex geodesics in $\mathcal{B}_{1}$ through $p$ to complex geodesics in $\mathcal{B}_{2}$ through $f(p)$ and, in particular, the linear map $d f_{p}$ sends complex lines in $T_{p} \mathcal{B}_{1}$ to complex lines in $T_{p} \mathcal{B}_{2}$. We conclude that $d f_{p}$ is either a complex linear map or complex anti-linear map.

The assumption that the Kobayashi metric of $\mathcal{B}_{1}$ and $\mathcal{B}_{2}$ is complete implies that there is a complex geodesic between any pair of distinct points in $\mathcal{B}_{1}$ and $\mathcal{B}_{2}$. Hence, $d f_{p}$ is either complex linear for almost every $p \in \mathcal{B}_{1}$ or complex anti-linear for almost every $p \in \mathcal{B}_{1}$. In particular, up to conjugation, $f$ is holomorphic as a distribution and the theorem follows from Weyl's regularity Lemma [15]. 
Open Access This article is distributed under the terms of the Creative Commons Attribution 4.0 International License (http://creativecommons.org/licenses/by/4.0/), which permits unrestricted use, distribution, and reproduction in any medium, provided you give appropriate credit to the original author(s) and the source, provide a link to the Creative Commons license, and indicate if changes were made.

\section{References}

1. Ahiezer, N.I., Ronkin, L.I.: Separately analytic functions of several variables, and "edge of the wedge" theorems. Uspehi Mat. Nauk 28, 27-42 (1973)

2. Baracco, L., Zampieri, G.: Separate real analyticity and CR extendibility. Forum Math. 21, 519-531 (2009)

3. Bers, L.: Finite dimensional Teichmüller spaces and generalizations. Bull. Am. Math. Soc. 5, 131-172 (1981)

4. Earle, C.J., Kra, I., Krushkal, S.L.: Holomorphic motions and Teichmüller spaces. Trans. Am. Math. Soc. 343, 927-948 (1994)

5. Earle, C.J., Li, Z.: Isometrically embedded polydisks in infinite dimensional Teichmüller spaces. J. Geom. Anal. 9, 51-71 (1999)

6. Farb, B., Margalit, D.: A Primer on Mapping Class Groups. Princeton University Press, Princeton (2012)

7. Federer, H.: Geometric Measure Theory. Springer, New York (1969)

8. Fletcher, A., Markovic, V.: Infinite dimensional Teichmüller spaces. In: Papadopoulos, A. (ed.) Handbook of Teichmüller Theory, vol. II, pp. 65-92. Eur. Math. Soc (2009)

9. Gardiner, F.P., Lakic, N.: Quasiconformal Teichmüller Theory. American Mathematical Society, New York (2000)

10. Harish, S., Hervé, G.: Totally geodesic discs in strongly convex domains. Math. Z. 274, 185-197 (2013)

11. Hörmander, L.: An introduction to complex analysis in several variables, volume 7 of North-Holland Mathematical Library, 3rd edn. North-Holland Publishing Co., Amsterdam (1990)

12. Hubbard, J.H.: Teichmüller Theory, vol. I. Matrix Editions, Ithaca (2006)

13. Jarnicki, M., Pflug, P.: Separately Analytic Functions. European Mathematical Society (EMS), Zürich (2011)

14. Kobayashi, S.: Hyperbolic Complex Spaces. Springer, New York (1998)

15. Krantz, S.G.: Function Theory of Several Complex Variables. Wiley, Amsterdam (1982)

16. McMullen, C.: From dynamics on surfaces to rational points on curves. Bull. Am. Math. Soc. 37, 119-140 (2000)

17. Nikolov, N., Pflug, P., Zwonek, W.: Estimates for invariant metrics on C-convex domains. Trans. Am. Math. Soc. 363, 6245-6256 (2011)

18. Ratcliffe, J.G.: Foundations of Hyperbolic Manifolds. Springer, New York (1994)

19. Royden, H.L.: Automorphisms and isometries of Teichmüller space. In: Advances in the Theory of Riemann Surfaces, pp. 369-384. Princeton University Press, Princeton (1971)

20. Słodkowski, Z.: Holomorphic motions and polynomial hulls. Proc. Am. Math. Soc. 111, 347-355 (1991)

21. Sullivan, D.: On the ergodic theory at infinity of an arbitrary discrete group of hyperbolic motions. In Kra, I., Maskit, B. (eds.), Riemann Surfaces and Related Topics: Proceedings of the 1978 Stony Brook Conference, volume 97 of Annals of Math. Studies. Princeton University Press, Princeton (1981)

22. Tanigawa, H.: Holomorphic mappings into Teichmüller spaces. Proc. Am. Math. Soc. 117, 71-78 (1993) 